\title{
SOLUTIONS OF THE DIVERGENCE AND ANALYSIS OF THE STOKES EQUATIONS IN PLANAR HÖLDER- $\alpha$ DOMAINS
}

\author{
RICARDO G. DURÁN AND FERNANDO LÓPEZ GARCÍA
}

\begin{abstract}
If $\Omega \subset \mathbb{R}^{n}$ is a bounded domain, the existence of solutions $\mathbf{u} \in H_{0}^{1}(\Omega)^{n}$ of $\operatorname{div} \mathbf{u}=f$ for $f \in L^{2}(\Omega)$ with vanishing mean value, is a basic result in the analysis of the Stokes equations. In particular it allows to show the existence of a solution $(\mathbf{u}, p) \in$ $H_{0}^{1}(\Omega)^{n} \times L^{2}(\Omega)$, where $\mathbf{u}$ is the velocity and $p$ the pressure.

It is known that the above mentioned result holds when $\Omega$ is a Lipschitz domain and that it is not valid for arbitrary Hölder- $\alpha$ domains.

In this paper we prove that if $\Omega$ is a planar simply connected Hölder- $\alpha$ domain, there exist solutions of $\operatorname{div} \mathbf{u}=f$ in appropriate weighted Sobolev spaces, where the weights are powers of the distance to the boundary. Moreover, we show that the powers of the distance in the results obtained are optimal.

For some particular domains with an external cusp we apply our results to show the well posedness of the Stokes equations in appropriate weighted Sobolev spaces obtaining as a consequence the existence of a solution $(\mathbf{u}, p) \in H_{0}^{1}(\Omega)^{n} \times L^{r}(\Omega)$ for some $r<2$ depending on the power of the cusp.
\end{abstract}

\section{INTRODUCTION}

Let $\Omega \in \mathbb{R}^{n}$ be a bounded open domain. We will use standard notations for Sobolev spaces and, for $1<p<\infty, L_{0}^{p}(\Omega)$ will denote the subspace of functions in $L^{p}(\Omega)$ with vanishing mean value.

The Stokes equations modeling the displacement of a viscous incompressible fluid contained in $\Omega$ are given by

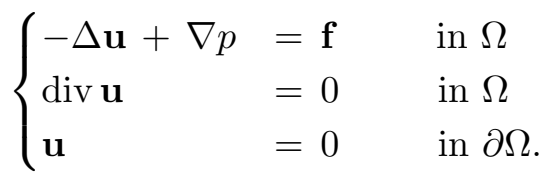

where $\mathbf{u}$ is the velocity and $p$ the pressure.

Variational analysis of this system of equations is fundamental for the development of finite element numerical approximations as well as for the study of their accuracy. Consequently, a lot of work has been done in this direction. However, the standard well known theory is based on the assumption that $\Omega$ is a Lipschitz domain. Our goal is to extend the variational analysis of the Stokes equations to a more general class of domains. With this purpose we will work with Hölder- $\alpha$ domains.

For Lipschitz domains it is known that, for $\mathbf{f} \in H^{-1}(\Omega)^{n}$, there exists a unique solution $(\mathbf{u}, p) \in H_{0}^{1}(\Omega)^{n} \times L_{0}^{2}(\Omega)$ of $(1.1)$ and moreover,

$$
\|\mathbf{u}\|_{H_{0}^{1}(\Omega)}+\|p\|_{L^{2}(\Omega)} \leq C\|\mathbf{f}\|_{H^{-1}(\Omega)},
$$

see for example [BS, BF, GR, T].

The basic result to prove this existence and uniqueness theorem is the following result on solutions of the divergence: If $\Omega$ is a Lipschitz domain, for any $f \in L_{0}^{2}(\Omega)$ there exists $\mathbf{u} \in H_{0}^{1}(\Omega)^{n}$ such that

1991 Mathematics Subject Classification. Primary: 26D10, 35Q30 ; Secondary 76D03.

Key words and phrases. Divergence Operator, planar Hölder- $\alpha$ domains, Stokes equations. 


$$
\operatorname{div} \mathbf{u}=f \quad \text { in } \Omega
$$

and

$$
\|\mathbf{u}\|_{H_{0}^{1}(\Omega)} \leq C\|f\|_{L^{2}(\Omega)}
$$

where the constant $C$ depends only on $\Omega$.

The existence of solutions of (1.2) satisfying (1.3) is also closely connected with the Korn inequality which is fundamental in the analysis of the elasticity equations (see [HP]).

Because of these applications, this problem, as well as it generalizations to the $L^{p}$ case, has been widely analyzed and several different arguments have been given to prove it under different assumptions on the domain. We refer the reader for example to $[\mathrm{ASV}, \mathrm{Br}, \mathrm{BB}, \mathrm{BS}$, $\mathrm{B}, \mathrm{GR}, \mathrm{DM} 2]$. Also, generalizations of this result to the case of fractional order Sobolev spaces have been proved recently in [CM, GHH].

Moreover, in $[\mathrm{ADM}]$, the existence of solutions of (1.2) satisfying

$$
\|\mathbf{u}\|_{W_{0}^{1, p}(\Omega)} \leq C\|f\|_{L^{p}(\Omega)},
$$

for $1<p<\infty$, was proved for the so called John domains, which form a large class containing properly the Lipschitz domains. For the particular case of planar simply connected domains, it is shown in $[\mathrm{ADM}]$ that being a John domain is also a necessary condition in the case $1<p<2$. In particular, there exist bounded domains and values of $p$ for which solutions of (1.2) satisfying (1.4) do not exist.

Actually, this fact was previously well known, indeed, several arguments have been given to show it. For example, in the old paper [Fr], Friedrichs proved that, for smooth planar domains, the $L^{2}$-norm of the conjugate of a harmonic function $f$ (normalized in an appropriate way) is bounded by the $L^{2}$-norm of $f$ times a constant depending only on the domain. Moreover, he showed that this inequality is not valid if the domain has an external cusp of quadratic type. It is easy to see that the Friedrichs inequality can be deduced from the existence of $\mathbf{u}$ satisfying (1.2) and (1.3). Therefore, such a $\mathbf{u}$ cannot exist for that kind of domains. More recently other examples have been given in $[\mathrm{GG}]$ and in an unpublished work of Gabriel Acosta. Acosta's examples are very elementary and applies to external cusps of power type with any power $\gamma>1$ and any $1<p<\infty$. We refer the reader to [Du1] where a particular case has been reproduced.

On the other hand, existence and regularity of solutions of elliptic equations in domains with external cusps have been considered by several authors. Let us mention for example [AADL, Da, MP1, MP2, MP3, N, NT]. However, as far as we know, there are no results for the Stokes equations on this kind of domains.

Therefore, it seems natural to ask whether the existence of solutions of (1.2) satisfying an estimate weaker than (1.3) can be proved for the above mentioned domains. Moreover, if this is the case, can these results be applied to show the well posedness of the Stokes problem in appropriate Hilbert spaces?

In this paper we give some partial answers to these questions in the particular case of planar domains. We consider simply connected planar Hölder $\alpha$ domains $\Omega$, with $0<\alpha \leq 1$, i. e., $\partial \Omega$ is locally the graph of a Hölder $\alpha$ function. For these domains we prove the existence of solutions of (1.2) satisfying weaker estimates than (1.4) involving weighted norms where the weights are powers of the distance to the boundary. For general Hölder $\alpha$ domains the zero boundary condition will be imposed in a weak way. Afterwards, in some particular examples, we will show that this weak boundary condition agrees with the usual one.

As an application we will prove the well posedness, in appropriate weighted norms, of the Stokes equations for some cuspidal domains. As a consequence we will obtain in particular that, for this kind of domains, given $\mathbf{f} \in H^{-1}(\Omega)^{2}$ there exists a unique solution $(\mathbf{u}, p) \in$ 
$H_{0}^{1}(\Omega)^{2} \times L_{0}^{r}(\Omega)$ of the Stokes equations (1.1) satisfying

$$
\|\mathbf{v}\|_{H_{0}^{1}(\Omega)}+\|p\|_{L^{r}(\Omega)} \leq C\|\mathbf{f}\|_{H^{-1}(\Omega)}
$$

for some $1<r \leq 2$ which depends on the power of the cusp.

Our approach use some of the ideas of the papers [ADL, GK]. The existence of solutions of the divergence is derived from appropriate Korn type inequalities. The weighted Korn inequalities that we need are slight variants of those obtained in [ADL] but we include the proofs for the sake of completeness.

Although our arguments to prove the existence of solutions of the divergence are two dimensional, we write the proofs of the Korn type inequalities in the general $n$-dimensional case because they have interest in themselves.

The rest of the paper is organized as follows. In Section 2 we introduce some notations and prove the weighted Korn inequalities. Section 3 deals with our main results concerning the existence of solutions of the divergence in appropriate weighted Sobolev spaces for Hölder $\alpha$ domains. In Section 4 we apply the results of the previous section for the particular case of domains having power type external cusps. We show that in this case our weak zero boundary condition agrees with the usual one. Also in this section we prove optimality of our results. In Section 5 we show how our results can be applied to prove the well posedness of the Stokes equations in appropriate Hilbert spaces.

\section{Preliminaries and Korn type inequalities}

Let $\Omega$ be a bounded open subset of $\mathbb{R}^{n}$ and $d(x)$ the distance of $x \in \Omega$ to the boundary $\partial \Omega$. We will denote by $L^{p}(\Omega, \gamma)$ the Banach space given by the norm

$$
\|u\|_{L^{p}(\Omega, \gamma)}:=\left\|u d^{\gamma}\right\|_{L^{p}(\Omega)}
$$

and, analogously, $W^{1, p}(\Omega, \gamma)$ will be the Banach space with norm

$$
\|u\|_{W^{1, p}(\Omega, \gamma)}:=\left\|u d^{\gamma}\right\|_{L^{p}(\Omega)}+\left\|\nabla u d^{\gamma}\right\|_{L^{p}(\Omega)} .
$$

Whenever $L^{p}(\Omega, \gamma) \subset L^{1}(\Omega)$ we will call $L_{0}^{p}(\Omega, \gamma)$ the subspace of $L^{p}(\Omega, \gamma)$ formed by functions of vanishing mean value. Since no confusion is possible we will use the same notations for the norms of vector or tensor fields.

For a vector field $\mathbf{u}=\left(u_{1}, \cdots, u_{n}\right)$ defined in $\Omega$ we denote by $D \mathbf{u}$ the jacobian matrix, namely, $(D \mathbf{u})_{i j}=\frac{\partial u_{i}}{\partial x_{j}}$ and by $\varepsilon(\mathbf{u})$ its symmetric part (i.e., the linear strain tensor associated with $\mathbf{u})$, that is $\varepsilon(\mathbf{u})_{i j}=\frac{1}{2}\left(\frac{\partial u_{i}}{\partial x_{j}}+\frac{\partial u_{j}}{\partial x_{i}}\right)$.

We start by giving a weighted Korn inequality for Hölder $\alpha$ domains. The statement given in the following theorem is slightly stronger than the result in Theorem 3.1 of [ADL]. Therefore, we include the proof for the sake of completeness although the arguments are essentially those given in that reference. In particular we will make use of the following improved Poincaré inequality proved in [ADL, Theorem 2.1]. If $\Omega$ is a Hölder $\alpha$ domain, $0<\alpha \leq 1, B \subset \Omega$ a ball and $\phi \in C_{0}^{\infty}(B)$ is such that $\int_{B} \phi=1$ then, for $\alpha \leq \beta \leq 1$ and $f$ such that $\int_{B} f \phi=0$ there exists a constant $C$ depending only on $\Omega, B$ and $\phi$ such that,

$$
\|f\|_{L^{p}(\Omega, 1-\beta)} \leq C\|\nabla f\|_{L^{p}(\Omega, 1+\alpha-\beta)} .
$$

Theorem 2.1. Let $\Omega \subset \mathbb{R}^{n}$ be a Hölder $\alpha$ domain, $B \subset \Omega$ a ball and $1<p<\infty$. Then, for $\alpha \leq \beta \leq 1$ the following inequality holds,

$$
\|D \mathbf{u}\|_{L^{p}(\Omega, 1-\beta)} \leq C\left\{\|\varepsilon(\mathbf{u})\|_{L^{p}(\Omega, \alpha-\beta)}+\|\mathbf{u}\|_{L^{p}(B)}\right\}
$$

where the constant $C$ depends only on $\Omega, B$ and $p$. 
Proof. Following $[\mathrm{KO}]$, we can show that there exists $\mathbf{v} \in W^{1, p}(\Omega)^{n}$ such that

$$
\Delta \mathbf{v}=\Delta \mathbf{u} \quad \text { in } \Omega
$$

and

$$
\|\mathbf{v}\|_{W^{1, p}(\Omega)} \leq C\|\varepsilon(\mathbf{u})\|_{L^{p}(\Omega)} .
$$

Now, let $\phi \in C_{0}^{\infty}(B)$ be such that $\int_{B} \phi d x=1$. For $i=1, \ldots, n$ define the linear functions

$$
L_{i}(x):=\left(\int_{B} \nabla\left(u_{i}-v_{i}\right) \phi\right) \cdot x
$$

and $\mathbf{L}(x)$ as the vector with components $L_{i}(x)$.

Then,

$$
D \mathbf{L}=\int_{B} D(\mathbf{u}-\mathbf{v}) \phi
$$

and, integrating by parts and applying the Hölder inequality we obtain

$$
|D \mathbf{L}| \leq\|\mathbf{u}-\mathbf{v}\|_{L^{p}(B)}\|\nabla \phi\|_{L^{p^{\prime}(B)}}
$$

where $p^{\prime}$ is the dual exponent of $p$.

Therefore, it follows from (2.4) that there exists a constant $C$ depending only on $\Omega, p$ and $\phi$ such that

Let us now introduce

$$
\|D \mathbf{L}\|_{L^{p}(\Omega)} \leq C\left\{\|\mathbf{u}\|_{L^{p}(B)}+\|\varepsilon(\mathbf{u})\|_{L^{p}(\Omega)}\right\} .
$$

$$
\mathbf{w}:=\mathbf{u}-\mathbf{v}-\mathbf{L} .
$$

Then, in view of the bounds (2.4) and (2.5), it only remains to estimate w. But, from (2.3) and the fact that $\mathbf{L}$ is linear we know that

$$
\Delta \mathbf{w}=0
$$

and consequently,

$$
\Delta \varepsilon_{i j}(\mathbf{w})=0 .
$$

But, if $f$ is a harmonic function in $\Omega$, the following estimate holds

$$
\|\nabla f\|_{L^{p}(\Omega, 1-\mu)} \leq C\|f\|_{L^{p}(\Omega,-\mu)}
$$

for all $\mu \in \mathbb{R}$. Indeed, this estimate was proved in [De] (see also Lema 3.1 in [ADL], and [KO] for a different proof in the case $p=2$ and $\mu=0$ ).

Therefore, taking $\mu=\beta-\alpha$ we obtain

$$
\left\|\nabla \varepsilon_{i j}(\mathbf{w})\right\|_{L^{p}(\Omega, 1+\alpha-\beta)} \leq C\left\|\varepsilon_{i j}(\mathbf{w})\right\|_{L^{p}(\Omega, \alpha-\beta)}
$$

and using the well known identity

we conclude that

$$
\frac{\partial^{2} w_{i}}{\partial x_{j} \partial x_{k}}=\frac{\partial \varepsilon_{i k}(\mathbf{w})}{\partial x_{j}}+\frac{\partial \varepsilon_{i j}(\mathbf{w})}{\partial x_{k}}-\frac{\partial \varepsilon_{j k}(\mathbf{w})}{\partial x_{i}}
$$

$$
\left\|\frac{\partial^{2} w_{i}}{\partial x_{j} \partial x_{k}}\right\|_{L^{p}(\Omega, 1+\alpha-\beta)} \leq C\|\varepsilon(\mathbf{w})\|_{L^{p}(\Omega, \alpha-\beta)}
$$

for any $i, j$ and $k$.

Since $\int \frac{\partial w_{i}}{\partial x_{j}} \phi=0$ (indeed, we have defined $\mathbf{L}$ in order to have this property), it follows from the improved Poincaré inequality (2.2) that

$$
\left\|\frac{\partial w_{i}}{\partial x_{j}}\right\|_{L^{p}(\Omega, 1-\beta)} \leq C\left\|\nabla \frac{\partial w_{i}}{\partial x_{j}}\right\|_{L^{p}(\Omega, 1+\alpha-\beta)} .
$$


Therefore, using (2.6), we obtain

$$
\|D \mathbf{w}\|_{L^{p}(\Omega, 1-\beta)} \leq C\|\varepsilon(\mathbf{w})\|_{L^{p}(\Omega, \alpha-\beta)} \leq C\|\varepsilon(\mathbf{u})\|_{L^{p}(\Omega, \alpha-\beta)}
$$

concluding the proof.

In the following corollary we give a weighted Korn inequality for Hölder $\alpha$ domains which can be seen as a generalization of the so-called second case of Korn inequality. To state this inequality we need to introduce the space of infinitesimal rigid motions, namely,

$$
\mathcal{N}=\left\{\mathbf{v} \in W^{1, p}(\Omega)^{n}: \varepsilon(\mathbf{v})=0\right\} .
$$

Corollary 2.1. Let $\Omega \subset \mathbb{R}^{n}$ be a Hölder $\alpha$ domain and $1<p<\infty$. Then, for $\alpha \leq \beta \leq 1$ the following inequality holds,

$$
\inf _{\mathbf{v} \in \mathcal{N}}\|\mathbf{u}-\mathbf{v}\|_{W^{1, p}(\Omega, 1-\beta)} \leq C\|\varepsilon(\mathbf{u})\|_{L^{p}(\Omega, \alpha-\beta)} .
$$

Proof. Take $B$ and $\phi$ as in the previous theorem with $\bar{B} \subset \Omega$. Define $\bar{x}_{i}=\int_{B} x_{i} \phi(x) d x$ and $\mathbf{v} \in W^{1, p}(\Omega)^{n}$ defined by

$$
v_{i}(x)=a_{i}+\sum_{j=1}^{n} b_{i j}\left(x_{j}-\bar{x}_{j}\right)
$$

with

$$
a_{i}=\int_{B} u_{i} \phi \quad \text { and } \quad b_{i j}=\frac{1}{2|B|} \int_{B}\left(\frac{\partial u_{i}}{\partial x_{j}}-\frac{\partial u_{j}}{\partial x_{i}}\right) .
$$

It is easy to check that $\mathbf{v} \in \mathcal{N}$. Now, since $\int_{B}(\mathbf{u}-\mathbf{v}) \phi=0$, it follows from (2.2) (actually we are using only a weaker standard Poincaré inequality with weights) and Theorem 2.1 that

$$
\|\mathbf{u}-\mathbf{v}\|_{W^{1, p}(\Omega, 1-\beta)} \leq C\left\{\|\varepsilon(\mathbf{u}-\mathbf{v})\|_{L^{p}(\Omega, \alpha-\beta)}+\|\mathbf{u}-\mathbf{v}\|_{L^{p}(B)}\right\}
$$

and using now the Poincaré inequality in $B$ we have

$$
\|\mathbf{u}-\mathbf{v}\|_{W^{1, p}(\Omega, 1-\beta)} \leq C\left\{\|\varepsilon(\mathbf{u}-\mathbf{v})\|_{L^{p}(\Omega, \alpha-\beta)}+\|D(\mathbf{u}-\mathbf{v})\|_{L^{p}(B)}\right\} .
$$

But,

$$
\int_{B}\left(\frac{\partial(u-v)_{i}}{\partial x_{j}}-\frac{\partial(u-v)_{j}}{\partial x_{i}}\right)=0
$$

and therefore, the so-called second case of Korn inequality applied in $B$ gives

$$
\|D(\mathbf{u}-\mathbf{v})\|_{L^{p}(B)} \leq C\|\varepsilon(\mathbf{u}-\mathbf{v})\|_{L^{p}(B)} .
$$

Using this inequality in (2.8) and that $\varepsilon(\mathbf{v})=0$ we obtain

$$
\|\mathbf{u}-\mathbf{v}\|_{W^{1, p}(\Omega, 1-\beta)} \leq C\left\{\|\varepsilon(\mathbf{u})\|_{L^{p}(\Omega, \alpha-\beta)}+\|\varepsilon(\mathbf{u})\|_{L^{p}(B)}\right\}
$$

which implies (2.7) because $\bar{B} \subset \Omega$.

Remark 2.1. It is possible to prove the above corollary directly, i.e., without using the Korn inequality in the ball $B$, by using a standard compactness argument. Indeed, assuming that (2.7) does not hold and using that $W^{1, p}(\Omega, 1-\beta)$ is compactly embedded in $L^{p}(\Omega, \gamma)$ for any $\gamma>(1-\beta-\alpha) / \alpha($ see $[\mathrm{KuOp}$, Theorem 19.11]) and Theorem 2.1 one obtains a contradiction. 


\section{Solutions of the Divergence in Hölder $\alpha$ DOMAins}

This section deals with solutions of divergence in planar simply connected Hölder $\alpha$ domains. In what follows we restrict ourselves to the case $n=2$.

For regular enough bounded domains $\Omega$ (for example Lipschitz) it is known that, if $f \in$ $L_{0}^{p}(\Omega), 1<p<\infty$, there exists $\mathbf{u} \in W_{0}^{1, p}(\Omega)^{2}$ such that

$$
\operatorname{div} \mathbf{u}=f
$$

and

$$
\|\mathbf{u}\|_{W_{0}^{1, p}(\Omega)} \leq C\|f\|_{L^{p}(\Omega)}
$$

where the constant $C$ depends only on $\Omega$ and $p$.

On the other hand, as we have mentioned in the introduction, it is known that for general Hölder $\alpha$ domains this result is not valid. Our main goal is to prove a similar result for this kind of domains but using weighted norms.

We will use the following notation. For a scalar function $\psi$ we write $\operatorname{curl} \psi=\left(\frac{\partial \psi}{\partial x_{2}},-\frac{\partial \psi}{\partial x_{1}}\right)$ and for a vector field $\Psi=\left(\psi_{1}, \psi_{2}\right)$, Curl $\Psi$ denotes the matrix which has $\operatorname{curl} \psi_{i}$ as it rows. Furthermore, if $\sigma \in L^{p}(\Omega)^{2 \times 2}$, Div $\sigma$ denotes the vector field with components obtained by taking the divergence of the rows of $\sigma$.

We will impose the boundary condition in a weak form. To explain this weak condition observe first that to solve the problem it is enough to find a solution $\mathbf{u}$ of (3.1) such that the restriction to $\partial \Omega$ of both components of $\mathbf{u}$ are constant (whenever the domain is such that this restriction makes sense). Of course, we should replace the estimate (3.2) by

$$
\|D \mathbf{u}\|_{L^{p}(\Omega)} \leq C\|f\|_{L^{p}(\Omega)} .
$$

Afterwards, (3.2) would follow by applying the Poincaré inequality to the solution obtained by adding an appropriate constant vector field to $\mathbf{u}$ in order to obtain the vanishing boundary condition.

Now, assume that $\Omega$ is a Lipschitz domain. Then, if $\psi \in W^{1, p}(\Omega)$ satisfies

$$
\int_{\Omega} \operatorname{curl} \psi \cdot \nabla \phi=0 \quad \forall \phi \in W^{1, p^{\prime}}(\Omega)
$$

it follows by integration by parts that

$$
\int_{\partial \Omega} \frac{\partial \psi}{\partial t} \phi=0 \quad \forall \phi \in W^{1, p^{\prime}}(\Omega)
$$

where $\frac{\partial \psi}{\partial t}$ indicates the tangential derivative of $\psi$. Therefore $\frac{\partial \psi}{\partial t}=0$ and then the restriction of $\psi$ to $\partial \Omega$ is constant.

For a general domain $\Omega$ the tangential derivative on the boundary might not even be defined and therefore (3.5) would not make sense. However, condition (3.4) is well defined in any domain and this is the condition that we will use. Therefore we introduce the space

$$
W_{\text {const }}^{1, p}(\Omega) \subset W^{1, p}(\Omega)
$$

defined by

$$
W_{\text {const }}^{1, p}(\Omega)=\left\{\psi \in W^{1, p}(\Omega): \int_{\Omega} \operatorname{curl} \psi \cdot \nabla \phi=0 \quad \forall \phi \in W^{1, p^{\prime}}(\Omega)\right\}
$$

and more generally, for any $\gamma \in \mathbb{R}$,

$$
W_{\text {const }}^{1, p}(\Omega, \gamma)=\left\{\psi \in W^{1, p}(\Omega, \gamma): \int_{\Omega} \operatorname{curl} \psi \cdot \nabla \phi=0 \quad \forall \phi \in W^{1, p^{\prime}}(\Omega,-\gamma)\right\} .
$$

The proof of the following lemma uses ideas introduced in [GK] with different goals.

For $1<p<\infty$ and $\gamma \in \mathbb{R}, L_{\text {sym }}^{p}(\Omega, \gamma)^{2 \times 2}$ denotes the subspace of symmetric tensors in $L^{p}(\Omega, \gamma)^{2 \times 2}$. 
Lemma 3.1. Let $\Omega \subset \mathbb{R}^{2}$ be a Hölder $\alpha$ domain and $\mathbf{u} \in W^{1, p}(\Omega, \beta-1)^{2}$, with $\alpha \leq \beta \leq 1$, such that $\int_{\Omega}$ div $\mathbf{u}=0$. Then, there exists $\sigma \in L_{\text {sym }}^{p}(\Omega, \beta-\alpha)^{2 \times 2}$ satisfying

$$
\int_{\Omega} \sigma: D \mathbf{w}=\int_{\Omega} \operatorname{Curl} \mathbf{u}: D \mathbf{w}, \quad \forall \mathbf{w} \in W^{1, p^{\prime}}(\Omega, \alpha-\beta)^{2}
$$

and

$$
\|\sigma\|_{L^{p}(\Omega, \beta-\alpha)^{2 \times 2}} \leq C\|\operatorname{Curl} \mathbf{u}\|_{L^{p}(\Omega, \beta-1)^{2 \times 2}} .
$$

Proof. Let $H \subset L_{\text {sym }}^{p^{\prime}}(\Omega, \alpha-\beta)^{2 \times 2}$ the subspace defined as

$$
H=\left\{\tau \in L_{\text {sym }}^{p^{\prime}}(\Omega, \alpha-\beta)^{2 \times 2}: \tau=\varepsilon(\mathbf{w}) \text { with } \mathbf{w} \in W^{1, p^{\prime}}(\Omega, \alpha-\beta)^{2}\right\} .
$$

Let us see that the application

$$
T: \varepsilon(\mathbf{w}) \mapsto \int_{\Omega} \operatorname{Curl} \mathbf{u}: D \mathbf{w}
$$

defines a continuous linear functional on $H$.

First of all observe that $T$ is well defined. Indeed, it is enough to check that the expression on the right of (3.6) vanishes whenever $\varepsilon(\mathbf{w})=0$. But, it is known that in that case $\mathbf{w}(x, y)=$ $(a-c y, b+c x)$ and therefore

$$
\int_{\Omega} \operatorname{Curl} \mathbf{u}: D \mathbf{w}=c \int_{\Omega} \operatorname{div} \mathbf{u}=0 .
$$

Now, we want to show that $T$ is continuous on $H$. Using again that $\int_{\Omega} C u r l \mathbf{u}: D \mathbf{v}=0$ if $\varepsilon(\mathbf{v})=0$ and applying Corollary 2.1 we have, for $\tau=\varepsilon(\mathbf{w}) \in H$,

$$
\begin{aligned}
|T(\tau)| & =\left|\int_{\Omega} \operatorname{Curl} \mathbf{u}: D \mathbf{w}\right| \\
& \leq\|\operatorname{Curl} \mathbf{u}\|_{L^{p}(\Omega, \beta-1)^{2 \times 2}} \inf _{\mathbf{v} \in \mathcal{N}}\|D(\mathbf{w}-\mathbf{v})\|_{L^{p^{\prime}}(\Omega, 1-\beta)^{2 \times 2}} \\
& \leq C\|\operatorname{Curl} \mathbf{u}\|_{L^{p}(\Omega, \beta-1)^{2 \times 2}}\|\varepsilon(\mathbf{w})\|_{L^{p^{\prime}}(\Omega, \alpha-\beta)^{2 \times 2}} \\
& =C\|\operatorname{Curl} \mathbf{u}\|_{L^{p}(\Omega, \beta-1)^{2 \times 2}}\|\tau\|_{L^{p^{\prime}}(\Omega, \alpha-\beta)^{2 \times 2}} .
\end{aligned}
$$

By the Hahn-Banach theorem the functional $T$ can be extended to $L_{s y m}^{p^{\prime}}(\Omega, \alpha-\beta)^{2 \times 2}$ and therefore, by the Riesz representation theorem, there exists $\sigma \in L_{\text {sym }}^{p}(\Omega, \beta-\alpha)^{2 \times 2}$ such that

and

$$
T(\tau)=\int_{\Omega} \sigma: \tau \quad \forall \tau \in L_{\text {sym }}^{p^{\prime}}(\Omega, \alpha-\beta)^{2 \times 2}
$$

$$
\|\sigma\|_{L^{p}(\Omega, \beta-\alpha)^{2 \times 2}} \leq C\|\operatorname{Curl} \mathbf{u}\|_{L^{p}(\Omega, \beta-1)^{2 \times 2}}
$$

where $C$ depends on the constant in Corollary 2.1. In particular,

$$
\int_{\Omega} \sigma: \varepsilon(\mathbf{w})=\int_{\Omega} \operatorname{Curl} \mathbf{u}: D \mathbf{w}
$$

for every $\mathbf{w} \in W^{1, p^{\prime}}(\Omega, \alpha-\beta)^{2}$. Then, we conclude the proof observing that, since $\sigma$ is symmetric, we can replace $\varepsilon(\mathbf{w})$ in $(3.7)$ by $D \mathbf{w}$.

It is a very well known result that a divergence free vector field is a rotational of a scalar function $\phi$. Indeed, for smooth vector fields the proof is usually given at elementary courses on calculus in several variables. On the other hand, if the vector field is only in $L^{p}(\Omega)^{2}$ but $\partial \Omega$ is Lipstchiz, it is not difficult to see that the vector field can be extended to a divergence free vector field defined in $\mathbb{R}^{2}$ and then, the existence of $\phi$ can be proved by using the Fourier transform. However, we need to use the existence of $\phi$ in the case where the domain and the 
vector field are both non-smooth. We have not been able to find a proof of this result in the literature and so we include the following lemma.

Lemma 3.2. Let $\Omega \subset \mathbb{R}^{2}$ be a simply connected Hölder $\alpha$ domain and $\alpha \leq \beta \leq 1$. Given a vector field $\mathbf{v} \in L^{p}(\Omega, 1-\beta)^{2}$ such that div $\mathbf{v}=0$, there exists $\phi \in W^{1, p}(\Omega, 1-\beta)$ such that

$$
\operatorname{curl} \phi=\mathbf{v} \quad \text { and } \quad\|\phi\|_{W^{1, p}(\Omega, 1-\beta)} \leq C\|\mathbf{v}\|_{L^{p}(\Omega, 1-\beta)}
$$

where $C$ is a constant depending only on $\Omega$.

Proof. Take $\psi \in C_{0}^{\infty}\left(B_{1}\right)$ satisfying $\int \psi=1$, where $B_{1}$ is the unit ball centered at the origin. For $k \geq 1$, define $\psi_{k}(x)=k^{2} \psi(k x)$ and, extending $\mathbf{v}$ by zero to $\mathbb{R}^{2}, \mathbf{v}_{k}=\psi_{k} * \mathbf{v}$.

Let $\Omega_{n}$ be a sequence of Lipschitz simply connected open subsets of $\Omega$ such that

$$
\bar{\Omega}_{n} \subset\left\{x \in \Omega: d(x)>\frac{1}{n}\right\} \quad \text { and } \quad \Omega_{n} \nearrow \Omega .
$$

Using that the distance between $\Omega_{n}$ and $\partial \Omega$ is greater than $1 / n$ and $\operatorname{supp} \psi_{k} \subset B\left(0, \frac{1}{k}\right)$, it is not difficult to see that $\operatorname{div} \mathbf{v}_{k}=0$ in $\Omega_{n}$ for every $k \geq n$.

Then, since $\mathbf{v}_{n} \in C_{0}^{\infty}\left(\mathbb{R}^{2}\right)^{2}$, there exists $\phi_{n} \in C_{0}^{\infty}\left(\Omega_{n}\right)$ such that $\operatorname{curl} \phi_{n}=\mathbf{v}_{n}$. Moreover, adding a constant we can take $\phi_{n}$ such that $\int_{\Omega_{1}} \phi_{n}=0$.

Now, by the Poincaré inequality we have, for any $n$, there exists a constant $C$ depending only on $n$ such that

$$
\left\|\phi_{k}-\phi_{k^{\prime}}\right\|_{L^{p}\left(\Omega_{n}\right)} \leq C\left\|\operatorname{curl}\left(\phi_{k}-\phi_{k^{\prime}}\right)\right\|_{L^{p}\left(\Omega_{n}\right)}=C\left\|\mathbf{v}_{k}-\mathbf{v}_{k^{\prime}}\right\|_{L^{p}\left(\Omega_{n}\right)} \rightarrow 0
$$

for $k, k^{\prime} \rightarrow \infty$.

Then, there exists $\phi \in L_{l o c}^{1}(\Omega)$ such that $\left.\phi_{k}\right|_{\Omega_{n}} \rightarrow \phi$ in $W^{1, p}\left(\Omega_{n}\right)$ and so $\operatorname{curl} \phi=\mathbf{v}$ in $\Omega_{n}, \forall n$ and consequently in $\Omega$.

Finally, using Theorem 2.1 of [ADL] we have

$$
\|\phi\|_{L^{p}(\Omega, 1-\beta)} \leq C\|\operatorname{curl} \phi\|_{L^{p}(\Omega, 1-\beta+\alpha)} \leq C\|\mathbf{v}\|_{L^{p}(\Omega, 1-\beta)}
$$

and the Lemma is proved.

We can now state and prove our results on solutions of the divergence on Hölder- $\alpha$ domains. As we mentioned above, it is known that for this kind of domains a solution of (3.1) satisfying (3.2) does not exist in general. Therefore, it is natural to look for solutions of (3.1) satisfying a weaker estimate. There are two possibilities: to use a stronger norm on the right of (3.2) or a weaker norm on the left. We will prove both kind of results but, to avoid technical complications while presenting the arguments, we give first a particular case of our results and postpone the generalization.

Theorem 3.1. Let $\Omega \subset \mathbb{R}^{2}$ be a bounded simply connected Hölder- $\alpha$ domain, $0<\alpha \leq 1$. Given $f \in L_{0}^{p}(\Omega), 1<p<\infty$, there exists $\mathbf{u} \in W_{\text {const }}^{1, p}(\Omega, 1-\alpha)^{2}$ such that

$$
\operatorname{div} \mathbf{u}=f
$$

and

$$
\|D \mathbf{u}\|_{L^{p}(\Omega, 1-\alpha)} \leq C\|f\|_{L^{p}(\Omega)}
$$

Proof. Take $\mathbf{v} \in W^{1, p}(\Omega)^{2}$ such that

$$
\operatorname{div} \mathbf{v}=f
$$

and

$$
\|\mathbf{v}\|_{W^{1, p}(\Omega)} \leq C\|f\|_{L^{p}(\Omega)} .
$$

The existence of such a $\mathbf{v}$ is well known, for example, since no boundary condition on $\mathbf{v}$ is required, we can extend $f$ by zero and take the solution of problem (3.1) and (3.2) in a ball containing $\Omega$. 
To prove the theorem it is enough to show that there exists $\mathbf{w} \in W^{1, p}(\Omega, 1-\alpha)^{2}$ satisfying $\operatorname{div} \mathbf{w}=0$ and such that

$$
\mathbf{v}-\mathbf{w} \in W_{\text {const }}^{1, p}(\Omega, 1-\alpha)^{2}
$$

and

$$
\|D \mathbf{w}\|_{L^{p}(\Omega, 1-\alpha)} \leq C\|f\|_{L^{p}(\Omega)} .
$$

Indeed, in view of (3.9), $\mathbf{u}:=\mathbf{v}-\mathbf{w}$ will be the desired solution.

But, since div $\mathbf{v}$ has vanishing mean value, we know from Lemma 3.1 that there exists $\sigma \in L_{\text {sym }}^{p}(\Omega, 1-\alpha)^{2 \times 2}$ satisfying

$$
\|\sigma\|_{L^{p}(\Omega, 1-\alpha)} \leq C\|C \operatorname{Curl} \mathbf{v}\|_{L^{p}(\Omega)}
$$

and

$$
\int_{\Omega} \sigma: D \mathbf{r}=\int_{\Omega} \operatorname{Curl} \mathbf{v}: D \mathbf{r} \quad, \quad \forall \mathbf{r} \in W^{1, p^{\prime}}(\Omega, \alpha-1)^{2}
$$

Then,

$$
\int_{\Omega} \operatorname{Div} \sigma \cdot \mathbf{r}=-\int_{\Omega} \sigma: D \mathbf{r}=-\int_{\Omega} \operatorname{Curl} \mathbf{v}: \operatorname{Dr}=\int_{\Omega} \operatorname{Div} \operatorname{Curl} \mathbf{v} \cdot \mathbf{r}=0
$$

for every $\mathbf{r} \in C_{0}^{\infty}(\Omega)^{2}$ and therefore $\operatorname{Div} \sigma=0$.

Now, from Lemma 3.2 we know that there exists $\mathbf{w} \in W^{1, p}(\Omega, 1-\alpha)^{2}$ such that

$$
\operatorname{Curl} \mathbf{w}=\sigma \quad \text { and } \quad\|\mathbf{w}\|_{W^{1, p}(\Omega, 1-\alpha)} \leq C\|\sigma\|_{L^{p}(\Omega, 1-\alpha)} .
$$

We have to check that $\operatorname{div} \mathbf{w}=0$, but since $\sigma$ is a symmetric tensor we have

$$
\operatorname{div} \mathbf{w}=\frac{\partial w_{1}}{\partial x_{1}}+\frac{\partial w_{2}}{\partial x_{2}}=-\sigma_{12}+\sigma_{21}=0 .
$$

To conclude the proof observe that in view of (3.10), (3.12) and (3.13) we have (3.11) which together with (3.10) yields (3.8).

Now, it is natural to ask whether part or all the weight in the estimate (3.8) can be moved to the right hand side. We will give a positive answer to this question. As we will show, the proof of this more general result is similar to that of Theorem 3.2 but it requires some non-trivial preliminary results. In particular, we will need an extra hypothesis on the domain.

We are going to use that some singular integral operators are continuous in weighted $L^{p_{-}}$ norms, $1<p<\infty$, for weights in the Muckenhoupt class $A_{p}$. This well known result as well as the definition of the $A_{p}$ classes can be seen for example in the book [S2].

In what follows we consider the distance to $\partial \Omega, d(x)$, defined for every $x \in \mathbb{R}^{n}$ and not only for $x \in \Omega$. We will give sufficient conditions on $\partial \Omega$ and on the exponent $\mu$ such that $d^{\mu}$ belongs to $A_{p}$. We state and prove the lemma in the more general situation of the distance to a compact set $F$ contained in $\mathbb{R}^{n}$ since this result can be of interest in other situations and its proof does not require any extra effort.

The following lemma generalizes the result proved in [DST] for smooth domains. Since the proof is too technical we postpone it for an appendix and continue now with our main results.

Given a compact set $F \subset \mathbb{R}^{n}$, we will denote with $d_{F}(x)$ the distance from $x$ to $F$.

Definition 3.1. For $0 \leq m \leq n$, a compact set $F \subset \mathbb{R}^{n}$ is an $\mathbf{m}$-regular set, if there exists a positive constant $C$ such that

$$
C^{-1} r^{m}<\mathcal{H}^{m}(B(x, r) \cap F)<C r^{m},
$$

for every $x \in F$ and $0<r \leq$ diam $F$, where $\mathcal{H}^{m}$ is the $m$-dimensional Hausdorff measure and $B(x, r)$ is the ball with radius $r$ and center $x$. The restriction $0<r \leq \operatorname{diam} F$ is eliminated if $F$ is a set with only one point. 
The reader who is not familiar with Hausdorff measure can think in the particular case that $F$ is a rectifiable curve in $\mathbb{R}^{2}$ and $m=1$ (this will be the case in the example of application to Stokes that we will give). In that case $\mathcal{H}^{1}$ is the length.

Lemma 3.3. Let $F \subset \mathbb{R}^{n}$ be a compact set included in a m-regular set $K$. If

$$
-(n-m)<\mu<(n-m)(p-1)
$$

then $d_{F}^{\mu}$ belongs to the class $A_{p}$.

Proof. See Apendix.

As a consequence we have the following result on weighted estimates for solutions of the divergence.

Lemma 3.4. Let $\Omega \subset \mathbb{R}^{2}$ be a bounded domain such that its boundary is contained in a 1-regular set. Given $f \in L^{p}(\Omega, \gamma), 1<p<\infty$, with $-1 / p<\gamma \leq 1-1 / p$ there exists $\mathbf{v} \in W^{1, p}(\Omega, \gamma)^{2}$ such that

$$
\operatorname{div} \mathbf{v}=f
$$

and

$$
\|\mathbf{v}\|_{W^{1, p}(\Omega, \gamma)^{2}} \leq C\|f\|_{L^{p}(\Omega, \gamma)}
$$

Proof. Extend $f$ by zero to $\mathbb{R}^{2}$. Then, it is well known that

$$
\phi(x)=-\frac{1}{2 \pi} \int_{\mathbb{R}^{n}} \log |x-y| f(y) d y
$$

is a solution of $\Delta \phi=f$. Moreover, it follows from the theory of singular integral operators (see for example [S2]) that, if $w \in A_{p}$,

$$
\int_{\mathbb{R}^{2}}\left|\frac{\partial^{2} \phi(x)}{\partial x_{i} \partial x_{j}}\right|^{p} w(x) d x \leq \int_{\mathbb{R}^{2}}|f(x)|^{p} w(x) d x .
$$

But, since $\mu=\gamma p$ satisfies the hypothesis of Lemma 3.3 with $n=2$ and $m=1, d^{\mu} \in A_{p}$ and therefore $\mathbf{v}:=\nabla \phi$ is the desired solution.

We can now give our more general result on solutions of the divergence.

Theorem 3.2. Let $\Omega \subset \mathbb{R}^{2}$ be a bounded domain such that its boundary is contained in a 1-regular set. Given $f \in L_{0}^{p}(\Omega, \beta-1), 1<p<\infty$, if $\alpha \leq \beta \leq 1$ and $-1 / p<\beta-1$, there exists $\mathbf{u} \in W_{\text {const }}^{1, p}(\Omega, \beta-\alpha)^{2}$ such that

$$
\operatorname{div} \mathbf{u}=f
$$

and

$$
\|D \mathbf{u}\|_{L^{p}(\Omega, \beta-\alpha)} \leq C\|f\|_{L^{p}(\Omega, \beta-1)}
$$

Proof. Since $-1 / p<\beta-1$, it follows from Lemma 3.4 that there exists $\mathbf{v} \in W^{1, p}(\Omega, \beta-1)^{2}$ such that

$$
\operatorname{div} \mathbf{v}=f
$$

and

$$
\|\mathbf{v}\|_{W^{1, p}(\Omega, \beta-1)} \leq C\|\mathbf{v}\|_{W^{1, p}(\Omega, \beta-\alpha)} \leq C\|f\|_{L^{p}(\Omega, \beta-1)} .
$$

The rest of the proof follows as that of Theorem 3.1. Now we have to show that there exists $\mathbf{w} \in W^{1, p}(\Omega, \beta-\alpha)^{2}$ satisfying $\operatorname{div} \mathbf{w}=0$ and such that

$$
\mathbf{v}-\mathbf{w} \in W_{\text {const }}^{1, p}(\Omega, \beta-\alpha)^{2}
$$

and

$$
\|D \mathbf{w}\|_{L^{p}(\Omega, \beta-\alpha)} \leq C\|f\|_{L^{p}(\Omega, \beta-1)} .
$$

The reader can easily check that the existence of $\mathbf{w}$ follows by using Lemma 3.1 as in Theorem 3.2 . 


\section{Domains with external Cusps}

In this section we consider the particular case of the Hölder- $\alpha$ domain defined as

$$
\Omega=\left\{(x, y) \in \mathbb{R}^{2}: 0<x<1,0<|y|<x^{1 / \alpha}\right\}
$$

with $0<\alpha \leq 1$.

We are going to show that in this case the weaker boundary condition imposed in Theorem 3.2 is equivalent to the standard one, i.e., that the solution of the divergence obtained in that theorem can be modified, by adding a constant vector field, to obtain a solution which vanishes on the boundary in the classic sense.

We will consider the particular case $\beta=\alpha$ of our general Theorem 3.2. Extension of the arguments to other cases might be possible but it is not straightforward.

Theorem 4.1. Let $\Omega \subset \mathbb{R}^{2}$ be the domain defined in (4.1) and $1<p<\infty$. If $1-1 / p<\alpha \leq 1$ then, given $f \in L_{0}^{p}(\Omega, \alpha-1)$ there exists $\mathbf{u} \in W_{0}^{1, p}(\Omega)^{2}$ such that

$$
\operatorname{div} \mathbf{u}=f
$$

and

$$
\|\mathbf{u}\|_{W_{0}^{1, p}(\Omega)} \leq C\|f\|_{L^{p}(\Omega, \alpha-1)}
$$

with a constant depending only on $\Omega, p$ and $\alpha$.

Proof. It is easy to see that $\Omega$ satisfies the hypotheses of Theorem 3.2. Therefore, it follows from that theorem that there exists $\mathbf{u} \in W_{\text {const }}^{1, p}(\Omega)^{2}$ which verifies (4.2). that

We are going to prove that, for any $\psi \in W_{\text {const }}^{1, p}(\Omega)$, there exists a constant $\psi_{0} \in \mathbb{R}$ such

$$
\psi-\psi_{0} \in W_{0}^{1, p}(\Omega):=\overline{C_{0}^{\infty}(\Omega)} .
$$

Consequently, $\mathbf{u}$ can be modified by adding a constant to each of its components to obtain the desired solution. Indeed, the estimate (4.3) will follow form (3.14) by the Poincaré inequality.

Given $\psi \in W_{\text {const }}^{1, p}(\Omega)$, let us show first that $\psi$ is constant on $\partial \Omega$. From the definition of $W_{\text {const }}^{1, p}(\Omega)$ we have that

$$
\int_{\Omega} \operatorname{curl} \psi \cdot \nabla \phi=0 \quad \forall \phi \in W^{1, p^{\prime}}(\Omega) .
$$

Now, let $\left(x_{0}, y_{0}\right)$ be a point in $\partial \Omega$ different from the origin and $B$ an open ball centered in $\left(x_{0}, y_{0}\right)$ such that $0 \notin B$. Taking $\phi \in C^{\infty}(B)$ we have

$$
0=\int_{\Omega} \operatorname{curl} \psi \cdot \nabla \phi=-\int_{B \cap \partial \Omega} \psi \frac{\partial \phi}{\partial t} \quad \forall \phi \in C^{\infty}(B)
$$

where $\frac{\partial \phi}{\partial t}$ indicates the tangential derivative of $\phi$. Consequently $\frac{\partial \psi}{\partial t}=0$ in the distributional sense on $B \cap \partial \Omega$ and then, since $\partial \Omega-(0,0)$ is a connected set, we conclude that there exists a constant $\psi_{0}$ such that $\psi=\psi_{0}$ on $\partial \Omega$. To simplify notation we assume in what follows that $\psi_{0}=0$ and so, we have to see that $\psi \in W_{0}^{1, p}(\Omega)$.

Now, let $\zeta \in C^{\infty}\left(\mathbb{R}_{+}\right)$be such that

$$
\zeta \equiv 1 \text { in }[0,1] \quad \zeta \equiv 0 \text { in } \mathbb{R}_{+}-(0,2) \quad 0 \leq \zeta \leq 1 .
$$

We decompose $\psi$ as

$$
\psi(x, y)=\zeta(3 x) \psi(x, y)+(1-\zeta(3 x)) \psi(x, y)=: \psi_{1}+\psi_{2} .
$$

It is easy to see that $\psi_{2} \in W_{0}^{1, p}\left(\Omega_{2}\right)$ where $\Omega_{2}$ is the Lipschitz domain

$$
\Omega_{2}:=\Omega \cap\left\{x>\frac{1}{3}\right\} .
$$


Thus, we can suppose that $\psi=\psi_{1}$. Let now $\phi_{n} \in C^{\infty}(\Omega)$ be a sequence satisfying $\phi_{n} \rightarrow \psi$ in $W^{1, p}(\Omega)$ and let $\gamma:=1 / \alpha$.

It is easy to check that, for $y \in(0,1)$,

$$
\left|\phi_{n}\left(x, x^{\gamma}-y\right)\right| \leq\left|\phi_{n}\left(x, x^{\gamma}\right)\right|+\int_{0}^{y}\left|\frac{\partial \phi_{n}}{\partial y}\left(x, x^{\gamma}-t\right)\right| d t .
$$

Therefore, integrating and using the Hölder inequality we have

$$
\int_{y^{\alpha}}^{1}\left|\phi_{n}\left(x, x^{\gamma}-y\right)\right|^{p} d x \leq C\left(\int_{y^{\alpha}}^{1}\left|\phi_{n}\left(x, x^{\gamma}\right)\right|^{p} d x+y^{p-1} \int_{y^{\alpha}}^{1} \int_{0}^{y}\left|\frac{\partial \phi_{n}}{\partial y}\left(x, x^{\gamma}-t\right)\right|^{p} d t d x\right) .
$$

Thus, using the continuity of the trace in the Lipschitz domain $\Omega \cap\left\{x>y^{\alpha}\right\}$ we have

$$
\begin{aligned}
\int_{y^{\alpha}}^{1}\left|\psi\left(x, x^{\gamma}-y\right)\right|^{p} d x & =\lim _{n \rightarrow \infty} \int_{y^{\alpha}}^{1}\left|\phi_{n}\left(x, x^{\gamma}-y\right)\right|^{p} d x \\
& \leq C \lim _{n \rightarrow \infty}\left(\int_{y^{\alpha}}^{1}\left|\phi_{n}\left(x, x^{\gamma}\right)\right|^{p} d x+y^{p-1} \int_{y^{\alpha}}^{1} \int_{0}^{y}\left|\frac{\partial \phi_{n}}{\partial y}\left(x, x^{\gamma}-t\right)\right|^{p} d t d x\right) \\
& =C y^{p-1} \int_{y^{\alpha}}^{1} \int_{0}^{y}\left|\frac{\partial \psi}{\partial y}\left(x, x^{\gamma}-t\right)\right|^{p} d t d x
\end{aligned}
$$

Now we will show that the sequence $\psi_{m}$ defined by

$$
\psi_{m}(x, y):=\psi(x, y)\left(1-\zeta_{m}\left(x^{\gamma}-|y|\right)\right)
$$

where $\zeta_{m}(t):=\zeta(m t)$, converges to $\psi$ in $W^{1, p}(\Omega)$. Moreover, it is easy to see that supp $\psi_{m} \subset$ $\Omega$.

By symmetry we can assume that $\Omega=\Omega \cap\{y>0\}$. Using the dominated convergence theorem we obtain

$$
\lim _{m \rightarrow \infty}\left\|\psi-\psi_{m}\right\|_{L^{p}(\Omega)}^{p}=\lim _{m \rightarrow \infty} \int_{\Omega}\left|\psi(x, y) \zeta_{m}\left(x^{\gamma}-y\right)\right|^{p}=0
$$

On the other hand,

$$
\frac{\partial \psi_{m}}{\partial x}(x, y)=\frac{\partial \psi}{\partial x}(x, y)\left(1-\zeta_{m}\left(x^{\gamma}-y\right)\right)-m \psi(x, y) \zeta^{\prime} \gamma x^{\gamma-1}
$$

and then,

$$
\begin{aligned}
\int_{\Omega}\left|\frac{\partial \psi}{\partial x}-\frac{\partial \psi_{m}}{\partial x}\right|^{p} & \leq \int_{\Omega}\left|\frac{\partial \psi}{\partial x}(x, y) \zeta_{m}\left(x^{\gamma}-y\right)\right|^{p}+C m^{p} \int_{\Omega}\left|\psi(x, y) \chi_{\{y>\psi(x)-2 / m\}}\right|^{p} \\
& =: I+I I .
\end{aligned}
$$

Thus, using again dominated convergence, it is easy to check that $I \rightarrow 0$. So, it only remains to analyze $I I$. 
Now, by the change of variables defined by $(x, y) \longmapsto\left(x, x^{\gamma}-y\right)$ and using (4.4) it follows that

$$
\begin{aligned}
I I & =C m^{p} \int_{0}^{2 / m} \int_{y^{\alpha}}^{1}\left|\psi\left(x, x^{\gamma}-y\right)\right|^{p} d x d y \\
& \leq C m^{p} \int_{0}^{2 / m} y^{p-1} \int_{y^{\alpha}}^{1} \int_{0}^{y}\left|\frac{\partial \psi}{\partial y}\left(x, x^{\gamma}-t\right)\right|^{p} d t d x d y \\
& \leq C m^{p} \int_{0}^{2 / m} y^{p-1} \int_{0}^{2 / m} \int_{t^{\alpha}}^{1}\left|\frac{\partial \psi}{\partial y}\left(x, x^{\gamma}-t\right)\right|^{p} d x d t d y \\
& \leq C m^{p}\left(\frac{2}{m}\right)^{p} \int_{0}^{2 / m} \int_{t^{\alpha}}^{1}\left|\frac{\partial \psi}{\partial y}\left(x, x^{\gamma}-t\right)\right|^{p} d x d t \\
& \leq C \int_{\Omega}\left|\frac{\partial \psi}{\partial y}(x, y) \chi_{\{y>\psi(x)-2 / m\}}\right|^{p} \longrightarrow 0
\end{aligned}
$$

An analogous argument can be applied to prove that $\frac{\partial \psi_{m}}{\partial y} \rightarrow \frac{\partial \psi}{\partial y}$ in $L^{p}(\Omega)$.

Consequently, we conclude the proof by observing that $\psi_{m}$ belongs to $W_{0}^{1, p}(\Omega)$.

In the following theorem we show that the estimate (4.3) is optimal in the sense that it is not possible to improve the power of the distance in the right hand side. Recall that $p^{\prime}=\frac{p}{p-1}$ is the dual exponent of $p$.

Theorem 4.2. Let $\Omega$ be the domain defined in (4.1). If for some $\beta \leq 0$ and for any $f \in L_{0}^{p}(\Omega, \beta)$ there exists $\mathbf{u} \in W_{0}^{1, p}(\Omega)^{2}$ such that

$$
\operatorname{div} \mathbf{u}=f
$$

and

$$
\|\mathbf{u}\|_{W_{0}^{1, p}(\Omega)} \leq C\|f\|_{L^{p}(\Omega, \beta)}
$$

with a constant $C$ independent of $f$ then, $\beta \leq \alpha-1$.

Proof. For $s<\frac{1-\beta p^{\prime}+\alpha}{\alpha p^{\prime}}$ define $f_{s}(x, y)=x^{-\frac{s}{p-1}} d(x, y)^{-p^{\prime} \beta}$. Then, calling $\Omega_{+}=\Omega \cap\{y>0\}$, we have

$$
\left\|f_{s}\right\|_{L^{p}(\Omega, \beta)}^{p}=2 \int_{\Omega_{+}} x^{-s p^{\prime}} d(x, y)^{-\beta p p^{\prime}+\beta p} d x d y=2 \int_{\Omega_{+}} x^{-s p^{\prime}} d(x, y)^{-\beta p^{\prime}} d x d y
$$

and therefore, using that for $y>0, d(x, y) \simeq x^{1 / \alpha}-y$, we obtain

$$
\left\|f_{s}\right\|_{L^{p}(\Omega, \beta)}^{p} \simeq 2 \int_{\Omega_{+}} x^{-s p^{\prime}}\left(x^{1 / \alpha}-y\right)^{-\beta p^{\prime}} d x d y
$$

but,

$$
\begin{gathered}
\int_{\Omega_{+}} x^{-s p^{\prime}}\left(x^{1 / \alpha}-y\right)^{-\beta p^{\prime}} d x d y=\int_{0}^{1} \int_{0}^{x^{\frac{1}{\alpha}}} x^{-s p^{\prime}}\left(x^{1 / \alpha}-y\right)^{-\beta p^{\prime}} d y d x \\
=\frac{1}{1-\beta p^{\prime}} \int_{0}^{1} x^{-s p^{\prime}} x^{\left(1-\beta p^{\prime}\right) / \alpha} d x=\frac{1}{1-\beta p^{\prime}} \frac{1}{p^{\prime}\left(\frac{1-\beta p^{\prime}+\alpha}{\alpha p^{\prime}}-s\right)}
\end{gathered}
$$

where we have used $s<\frac{1-\beta p^{\prime}+\alpha}{\alpha p^{\prime}}$. Therefore,

$$
\left\|f_{s}\right\|_{L^{p}(\Omega, \beta)}^{p} \simeq \frac{1}{A-s}
$$

where $A:=\frac{1-\beta p^{\prime}+\alpha}{\alpha p^{\prime}}$ and with constants in the equivalence independent of $s$. 
Now, let $B$ be a ball such that $\bar{B} \subset \Omega$ and $\omega \in C_{0}^{\infty}(B)$ such that $\int_{B} \omega=1$. From our hypothesis we know that, if $c_{s}=\int_{\Omega} f_{s}$, there exists $\mathbf{v}_{s} \in W_{0}^{1, p}(\Omega)^{2}$ such that

$$
\operatorname{div} \mathbf{v}_{s}=f_{s}-c_{s} \omega \quad \text { and } \quad\left\|\mathbf{v}_{s}\right\|_{W^{1, p}(\Omega)} \leq C\left\|f_{s}-c_{s} \omega\right\|_{L^{p}(\Omega, \beta)} .
$$

But, since $\beta \leq 0$,

$$
\left|c_{s}\right|=\left\|f_{s}\right\|_{L^{1}(\Omega)} \leq C\left\|f_{s}\right\|_{L^{p}(\Omega, \beta)}
$$

and so,

$$
\left\|\mathbf{v}_{s}\right\|_{W^{1, p}(\Omega)} \leq C\left\|f_{s}\right\|_{L^{p}(\Omega, \beta)}
$$

where we have used that $\|\omega\|_{L^{p}(\Omega, \beta)} \leq C$ because the support of $\omega$ is contained in $B$. Then,

$$
\begin{aligned}
\left\|f_{s}\right\|_{L^{p}(\Omega, \beta)}^{p} & =\int_{\Omega} f_{s}^{p-1}\left(f_{s}-c_{s} \omega\right) d^{p \beta}+\int_{\Omega} f_{s}^{p-1} c_{s} \omega d^{p \beta} \\
& =\int_{\Omega} f_{s}^{p-1} \operatorname{div} \mathbf{v}_{s} d^{p \beta}+\int_{\Omega} f_{s}^{p-1} c_{s} \omega d^{p \beta} \\
& =\int_{\Omega} x^{-s} \operatorname{div} \mathbf{v}_{s}+\int_{\Omega} f_{s}^{p-1} c_{s} \omega d^{p \beta} .
\end{aligned}
$$

Using again that the support of $\omega$ is at a positive distance from the boundary, together with (4.6), it follows that

$$
\int_{\Omega} f_{s}^{p-1} c_{s} \omega d^{p \beta} \leq C\left\|f_{s}\right\|_{L^{p}(\Omega, \beta)}
$$

On the other hand,

$$
\begin{aligned}
\int_{\Omega} x^{-s} \operatorname{div} \mathbf{v}_{s} & =s \int_{\Omega} x^{-s-1} \mathbf{v}_{s, 1}=s \int_{\Omega} \frac{\partial\left(y x^{-s-1}\right)}{\partial y} \mathbf{v}_{s, 1} \\
& =-s \int_{\Omega} y x^{-s-1} \frac{\partial \mathbf{v}_{s, 1}}{\partial y} \leq s\left\|y x^{-s-1}\right\|_{L^{p^{\prime}}(\Omega)}\left\|\mathbf{v}_{s}\right\|_{W^{1, p}(\Omega)} \\
& \leq C s\left\|y x^{-s-1}\right\|_{L^{p^{\prime}}(\Omega)}\left\|f_{s}\right\|_{L^{p}(\Omega, \beta)}
\end{aligned}
$$

where for the last inequality we have used (4.7).

Therefore,

$$
\left\|f_{s}\right\|_{L^{p}(\Omega, \beta)}^{p-1} \leq C\left\{s\left\|y x^{-s-1}\right\|_{L^{p^{\prime}}(\Omega)}+1\right\}
$$

But, an elementary computation shows that

$$
\left\|y x^{-s-1}\right\|_{L^{p^{\prime}(\Omega)}}^{p^{\prime}} \simeq \frac{1}{B-s}
$$

where $B:=\frac{1-(\alpha-1) p^{\prime}+\alpha}{\alpha p^{\prime}}$ and with constants in the equivalence independent of $s$.

Thus, from (4.5), (4.8) and (4.9) we conclude that there exists a constant independent of $s$ such that

$$
\frac{1}{A-s} \leq C \frac{1}{B-s}
$$

therefore, $B \leq A$ and it follows immediately that $\beta \leq \alpha-1$.

Remark 4.1. If we put some part of the weight in the left hand side as in (3.14), it is possible to prove a more general result, namely, under some restriction on the exponents in the weights, the difference between the powers in the right and left sides cannot be less than $1-\alpha$ (see [?]). 


\section{An application to the Stokes equations}

In this section we show how our results can be applied to the analysis of the Stokes equations when $\Omega$ is the domain defined in (4.1).

We are going to use the well known theory developed by Brezzi (see for example [BF, Du2, GR]) but modifying the usual Hilbert spaces and the bilinear form corresponding to the divergence free restriction in the weak formulation of the Stokes equations.

Theorem 5.1. Let $\Omega$ be the domain defined in (4.1) with $1 / 2<\alpha \leq 1$. Then, if $\mathbf{f} \in H^{-1}(\Omega)^{2}$, there exists a unique weak solution $(u, p) \in H_{0}^{1}(\Omega)^{2} \times L_{0}^{2}(\Omega, 1-\alpha)$ of the Stokes equations (1.1). Moreover, there exists a constant $C$ depending only on $\alpha$ such that

$$
\|\mathbf{v}\|_{H_{0}^{1}(\Omega)}+\|p\|_{L^{2}(\Omega, 1-\alpha)} \leq C\|\mathbf{f}\|_{H^{-1}(\Omega)} .
$$

Proof. Let us introduce the spaces

$$
V=\left\{\mathbf{v} \in H_{0}^{1}(\Omega)^{2}: \operatorname{div} \mathbf{v} \in L^{2}(\Omega, \alpha-1)\right\}
$$

which is a Hilbert space with the norm

$$
\|\mathbf{v}\|_{V}^{2}:=\|\mathbf{v}\|_{H_{0}^{1}(\Omega)}^{2}+\|\operatorname{div} \mathbf{v}\|_{L^{2}(\Omega, \alpha-1)}^{2},
$$

and

$$
Q=L_{0}^{2}(\Omega, \alpha-1) .
$$

Define the bilinear forms $a: V \times V \rightarrow \mathbb{R}$ and $b: V \times Q \rightarrow \mathbb{R}$ by

$$
a(\mathbf{u}, \mathbf{v})=\int_{\Omega} D \mathbf{u}: D \mathbf{v}
$$

and

$$
b(\mathbf{v}, q)=\int_{\Omega} \operatorname{div} \mathbf{v} q d^{2 \alpha-2} .
$$

We are going to show that the problem

$$
\begin{aligned}
& a(\mathbf{u}, \mathbf{v})-b(\mathbf{v}, q)=\int_{\Omega} \mathbf{f} \cdot \mathbf{v} \quad \forall \mathbf{v} \in V \\
& b(\mathbf{u}, r) \quad=0 \quad \forall r \in Q
\end{aligned}
$$

has a unique solution $(\mathbf{u}, q) \in V \times Q$.

Using the Schwarz inequality it is easy to check that the bilinear forms $a$ and $b$ are continuous and, since $\mathbf{f} \in H^{-1}(\Omega)^{2}$, that the linear functional defined by the right hand side of (5.2) is continuous.

Let

$$
W=\{\mathbf{v} \in V: b(\mathbf{v}, r)=0 \forall r \in Q\} .
$$

According to Brezzi's theory it is enough to see that $a$ is coercive in $W$ and $b$ satisfies the inf-sup condition

$$
\inf _{r \in Q} \sup _{\mathbf{v} \in V} \frac{b(\mathbf{v}, r)}{\|r\|_{Q}\|\mathbf{v}\|_{V}}>0
$$

Since $\operatorname{div} V \subset Q$ we can take $r=\operatorname{div} \mathbf{v}$ in the equation $b(\mathbf{v}, r)=0$ and conclude that $W=\left\{\mathbf{v} \in H_{0}^{1}(\Omega)^{2}: \operatorname{div} \mathbf{v}=0\right\}$. Therefore, coerciveness of $a$ in $W$ follows from the Poincaré inequality.

On the other hand, from Theorem 4.1 we know that given $r \in L_{0}^{2}(\Omega, \alpha-1)$ there exists $\mathbf{w} \in H_{0}^{1}(\Omega)$ such that

$$
\operatorname{div} \mathbf{w}=r \quad \text { and } \quad\|\mathbf{w}\|_{H_{0}^{1}(\Omega)} \leq C\|r\|_{L_{0}^{2}(\Omega, \alpha-1)}
$$


where $C$ is a positive constant which depends only on $\alpha$. Moreover, from the definition of the norm in $V$ it follows immediately that

$$
\|\mathbf{w}\|_{V} \leq C_{1}\|r\|_{Q}
$$

for another constant depending only on $\alpha$. Then,

$$
\sup _{\mathbf{v} \in V} \frac{b(\mathbf{v}, r)}{\|r\|_{Q}\|\mathbf{v}\|_{V}} \geq \frac{\int_{\Omega} \operatorname{div} \mathbf{w} r d^{2 \alpha-2}}{\|r\|_{Q}\|\mathbf{w}\|_{V}}=\frac{\|r\|_{Q}}{\|\mathbf{w}\|_{V}} \geq C_{1}^{-1}
$$

and therefore the inf-sup condition (5.4) is proved.

Summing up we have proved that the problem given in (5.2) and (5.3) has a unique solution $(\mathbf{u}, q) \in V \times Q$. Moreover, it follows also from the general theory that there exists a constant $C$ depending only on $C_{1}$ such that

$$
\|\mathbf{v}\|_{V}+\|q\|_{Q} \leq C\|\mathbf{f}\|_{H^{-1}(\Omega)} .
$$

Now, define $p=q d^{2 \alpha-2}$. It is easy to see that $p \in L^{2}(\Omega, 1-\alpha)$ and moreover, it follows from (5.3) that $\operatorname{div} \mathbf{u}=0$ and from (5.2) that (u,p) verifies

$$
\int_{\Omega} D \mathbf{u}: D \mathbf{v}-\int_{\Omega} \operatorname{div} \mathbf{v} p=\int_{\Omega} \mathbf{f} \cdot \mathbf{v} \quad \forall \mathbf{v} \in V
$$

Therefore, since $C_{0}^{\infty}(\Omega) \subset V,(\mathbf{u}, p)$ is a solution of the Stokes equations (1.1) in the sense of distributions as we wanted to prove. Finally, since $\|p\|_{L^{2}(\Omega, 1-\alpha)}=\|q\|_{Q}$, (5.1) follows immediately from (5.5).

We end this section with a corollary which gives an estimate for the pressure in a standard $L^{r}$-norm.

Corollary 5.1. Let $\Omega$ be the domain defined in (4.1) with $1 / 2<\alpha \leq 1$ and $(\mathbf{u}, p) \in H_{0}^{1}(\Omega)^{2} \times$ $L_{0}^{2}(\Omega, 1-\alpha)$ be the solution of the Stokes equations (1.1). If $\mathbf{f} \in H^{-1}(\Omega)^{2}$ and $1 \leq r<$ $2 /(3-2 \alpha)$ then $(\mathbf{u}, p) \in H_{0}^{1}(\Omega)^{2} \times L^{r}(\Omega)$. Moreover, there exists a constant $C$ depending only on $\alpha$ such that

$$
\|\mathbf{u}\|_{H_{0}^{1}(\Omega)}+\|p\|_{L^{r}(\Omega)} \leq C\|\mathbf{f}\|_{H^{-1}(\Omega)}
$$

Proof. We only have to prove that $p \in L^{r}(\Omega)$ and that

$$
\|p\|_{L^{r}(\Omega)} \leq C\|\mathbf{f}\|_{H^{-1}(\Omega)} .
$$

Observe that $\int_{o} d^{\beta}<+\infty$ for any $\beta>-1$. Indeed, this follows easily by using that $d(x, y) \simeq x^{1 / \alpha}-|y|$. Then, applying the Hölder inequality with exponent $2 / r$, we have

$$
\|p\|_{L^{r}(\Omega)}^{r}=\int_{\Omega}|p|^{r} d^{(1-\alpha) r} d^{(\alpha-1) r} \leq\|p\|_{L^{2}(\Omega, 1-\alpha)}^{r}\left(\int_{\Omega} d^{\frac{2(\alpha-1) r}{2-r}}\right)^{\frac{2-r}{2}}
$$

but the integral in the right hand side is finite because $(2(\alpha-1) r) /(2-r)>-1$. So $\|p\|_{L^{r}(\Omega)} \leq C\|p\|_{L^{2}(\Omega, 1-\alpha)}$ and therefore, (5.6) follows immediately from (5.1).

\section{Appendix}

To prove Lemma 3.3 we will work with Whitney decompositions. If $F$ is a compact nonempty subset of $\mathbb{R}^{n}$, then $\mathbb{R}^{n} \backslash F$ can be represented as a union of closed dyadic cubes with pairwise disjoint interior $Q_{j}^{k}$ satisfying

$$
\mathbb{R}^{n} \backslash F=\bigcup_{k \in \mathbb{Z}} \bigcup_{j=1}^{N_{k}} Q_{j}^{k}
$$


where the edge length of $Q_{j}^{k}$ is $2^{-k}$. The decomposition (6.1) is called a Whitney decomposition of $\mathbb{R}^{n} \backslash F$ and the collection $\left\{Q_{j}^{k}: j=1, \ldots, N_{k}\right\}$ is called the $k^{\text {th }}$ generation of Whitney cubes. Furthermore, the Whitney cubes satisfy

$$
\ell_{k} \leq d\left(Q_{j}^{k}, F\right) \leq 4 \ell_{k}
$$

where $d\left(Q_{j}^{k}, F\right)$ denotes the distance of the cube to $F$ and $\ell_{k}$ the diameter of $Q_{j}^{k}$ (see for example $[\mathrm{S}])$.

For $x_{0} \in F$ and $R>0, N_{k}\left(B\left(x_{0}, R\right)\right)$ denotes the number of Whitney cubes of $F^{c}$ in the $k^{\text {th }}$ generation contained in $B\left(x_{0}, R\right)$.

Lemma 6.1. Let $F \subset \mathbb{R}^{n}$ be a compact set included in an $\mathbf{m}$-regular set $K$. Given $x_{0} \in F$ and $0<R<\operatorname{diam} F / 3$, there exists a constant $C$ depending only on $K$ such that

$$
N_{k}\left(B\left(x_{0}, R\right)\right) \leq C R^{m} 2^{k m}
$$

Proof. We can assume that $2^{-k} \leq R$, if not $N_{k}\left(B\left(x_{0}, R\right)=0\right.$. The number of Whitney cubes of $F^{c}$ in the $k^{\text {th }}$ generation contained in the ball $B\left(x_{0}, R\right)$ can be estimated in terms of the number of balls of radius $2^{-k}$ and center contained in $F$ necessary to cover $F \cap B\left(x_{0}, 2 R\right)$. Indeed, suppose there exist balls $B\left(x_{i}, 2^{-k}\right)$ with $x_{i} \in F, 1 \leq i \leq N$, such that

$$
F \cap B\left(x_{0}, 2 R\right) \subseteq \bigcup_{i=1}^{N} B\left(x_{i}, 2^{-k}\right)
$$

and let $Q^{k}$ be a Whitney cube in the $k^{t h}$ generation contained in $B\left(x_{0}, R\right)$. Then, it is easy to check that

$$
d\left(Q^{k}, F\right)=d\left(Q^{k}, F \cap B\left(x_{0}, 2 R\right)\right) .
$$

Thus, if $y_{Q} \in F$ is a point satisfying $d\left(Q^{k}, F\right)=d\left(Q^{k}, y_{Q}\right)$, there exists some $i, 1 \leq i \leq N$, such that $y_{Q} \in B\left(x_{i}, 2^{-k}\right)$. So, using that $Q^{k}$ is a Whitney cube in the $k^{t h}$ generation it follows that

$$
Q^{k} \subset B\left(x_{i}, 6 \ell_{k}\right)
$$

But, $B\left(x_{i}, 6 \ell_{k}\right)$ cannot contain more than a finite number $c(n)$ of Whitney cubes $Q^{k}$. Then, by (6.2) it follows that

$$
N_{k}\left(B\left(x_{0}, R\right)\right) \leq c(n) N
$$

Therefore, to complete the proof we have to show that there exists $N$ balls satisfying (6.2) with $N \leq C R^{m} 2^{k m}$.

Let $r=2^{-(k+1)}$. For $K_{0}:=K \cap B\left(x_{0}, 2 R\right)$ we define the numbers

$$
H_{m}\left(K_{0}, r\right):=\min \left\{N r^{m}: K_{0} \subseteq \bigcup_{i=1}^{N} B\left(x_{i}, r\right), \text { with } x_{i} \in K_{0}\right\}
$$

and

$P\left(K_{0}, r\right):=\max \left\{N\right.$ : there exists disjoint balls $B\left(x_{i}, r\right), i=1, \ldots, N$, with $\left.x_{i} \in K_{0}\right\}$.

Then, using that $K$ is an m-regular set we have

$$
\begin{aligned}
H_{m}\left(K_{0}, r\right) & \leq P\left(K_{0}, \frac{r}{2}\right) r^{m}=2^{m} P\left(K_{0}, \frac{r}{2}\right)\left(\frac{r}{2}\right)^{m} \\
& <2^{m} C \sum_{i=1}^{P\left(K_{0}, r / 2\right)} \mathcal{H}^{m}\left(B\left(x_{i}, \frac{r}{2}\right) \cap K\right) \\
& =2^{m} C \sum_{i=1}^{P\left(K_{0}, r / 2\right)} \mathcal{H}^{m}\left(B\left(x_{i}, \frac{r}{2}\right) \cap K \cap B\left(x_{0}, 3 R\right)\right) \\
& \leq 2^{m} C \mathcal{H}^{m}\left(K \cap B\left(x_{0}, 3 R\right)\right)<C^{2} 6^{m} R^{m} .
\end{aligned}
$$


Thus, using the definition of $H_{m}\left(K_{0}, r\right)$ we obtain

$$
K \cap B\left(x_{0}, 2 R\right) \subseteq \bigcup_{i=1}^{N} B\left(x_{i}, 2^{-(k+1)}\right) \quad \text { and } \quad N \leq C R^{m} 2^{(k+1) m} .
$$

Now, $F$ is contained in $K$ and therefore it is possible to cover $F \cap B\left(x_{0}, 2 R\right)$ with $\bigcup_{i=1}^{N} B\left(x_{i}, 2^{-(k+1)}\right)$. Then, if $B\left(x_{i}, 2^{-(k+1)}\right)$ intersects $F$, for $x_{i}^{\prime} \in F \cap B\left(x_{i}, 2^{-(k+1)}\right)$ we have that

$$
B\left(x_{i}, 2^{-(k+1)}\right) \subseteq B\left(x_{i}^{\prime}, 2^{-k}\right) .
$$

Thus, it is easy to see that the balls $B\left(x_{i}^{\prime}, 2^{-k}\right)$ satisfy (6.2), concluding the proof.

Before proving Lemma 3.3 let us recall the definition of the Muckenhoupt class $A_{p}$. For $1<p<\infty$ a non-negative function $w$ is in $A_{p}$ if

$$
\sup _{B \subset \mathbb{R}^{n}}\left(\frac{1}{|B|} \int_{B} w(x) d x\right)\left(\frac{1}{|B|} \int_{B} w(x)^{-\frac{1}{p-1}} d x\right)^{p-1}<\infty .
$$

where the supremum is taken over all the balls $B$.

Proof of Lemma 3.3: Let $B$ be a ball in $\mathbb{R}^{n}, r_{B}$ its radius and $d(B)$ the distance of $B$ to F.

If $r_{B} \leq d(B)$, given $x$ in $B$ we have $d(B) \leq d(x) \leq 3 d(B)$. Then,

$$
\left(\frac{1}{|B|} \int_{B} d^{\mu}\right)\left(\frac{1}{|B|} \int_{B} d^{-\frac{\mu}{p-1}}\right)^{p-1} \leq C\left(\frac{1}{|B|} \int_{B} d(B)^{\mu}\right)\left(\frac{1}{|B|} \int_{B} d(B)^{-\frac{\mu}{p-1}}\right)^{p-1} \leq C
$$

On the other hand, if $r_{B} \geq d(B)$, there exists $x_{0} \in \partial \Omega$ such that $B \subseteq B\left(x_{0}, 3 r_{B}\right)$. Then, without loss of generality, we can assume that $B$ is centered at a point in $F$.

We consider two cases:

a) If $r_{B}<\operatorname{diam} F / 6$, from the Whitney decomposition of $F$ we have

$$
\left(\frac{1}{|B|} \int_{B} d^{\mu}\right)\left(\frac{1}{|B|} \int_{B} d^{-\frac{\mu}{p-1}}\right)^{p-1} \leq C r_{B}^{-n p}\left(\sum_{Q^{k}} \int_{Q^{k}} d^{\mu}\right)\left(\sum_{Q^{k}} \int_{Q^{k}} d^{-\frac{\mu}{p-1}}\right)^{p-1}=: \mathrm{I}
$$

where the sum is taken over all Whitney cubes $Q^{k}$ intersecting $B$. But it is enough to consider the Whitney cubes contained in the ball $2 B$.

Observe that if $Q^{k}$ is contained in $2 B$ then the diagonal of $Q^{k}$ is smaller than the diagonal of $2 B$. So, $2^{-k} \leq \frac{4}{\sqrt{n}} r_{B}$. Thus, if we call $k_{0}$ the minimum $k$ such that there exists $Q^{k}$ contained in $B$, it satisfies that $2^{-k_{0}} \leq C r_{B}$.

Now, using that $d(x) \simeq d\left(Q^{k}\right) \simeq 2^{-k}$ for every $x \in Q^{k}$ and Lemma 6.1 we obtain

$$
\begin{aligned}
& \leq C r_{B}^{-n p}\left(\sum_{Q_{k}} 2^{-k \mu} 2^{-k n}\right)\left(\sum_{Q_{k}} 2^{\frac{\mu k}{p-1}} 2^{-k n}\right)^{p-1} \\
& \leq C r_{B}^{-n p}\left(\sum_{k=k_{0}}^{\infty} N_{k}\left(B\left(x_{0}, 2 r_{B}\right)\right) 2^{-k \mu} 2^{-k n}\right)\left(\sum_{k=k_{0}}^{\infty} N_{k}\left(B\left(x_{0}, 2 r_{B}\right)\right) 2^{\frac{\mu k}{p-1}} 2^{-k n}\right)^{p-1} \\
& \leq C r_{B}^{-n p}\left(\sum_{k=k_{0}}^{\infty} r_{B}^{m} 2^{-k(\mu+n-m)}\right)\left(\sum_{k=k_{0}}^{\infty} r_{B}^{m} 2^{-k\left(n-m-\frac{\mu}{p-1}\right)}\right)^{p-1}=\mathrm{II} .
\end{aligned}
$$

Then, since $-(n-m)<\mu<(p-1)(n-m)$, we obtain 


$$
\mathrm{II} \leq C r_{B}^{-p(n-m)}\left(2^{-k_{0}(\mu+n-m)}\right)\left(2^{-k_{0}\left(n-m-\frac{\mu}{p-1}\right)}\right)^{p-1} \leq C r_{B}^{-p(n-m)}\left(2^{-k_{0}}\right)^{p(n-m)} \leq C
$$

b) If $r_{B} \geq \operatorname{diam} F / 6$, let $x_{F}$ be a point in $F$ independent of $B$. Then, since $x_{0} \in F$ and $r_{B}>\operatorname{diam} F / 6$ we can assume that $B$ is the ball with radius $r_{B}>3 \operatorname{diam} F$ and center $x_{F}$. On the other hand, if $B_{1}$ denotes the ball of radius 2 diam $K$ centered at $x_{F}$ we can see that $d^{\mu}$ and $d^{-\frac{\mu}{p-1}}$ are locally integrable. Then,

$$
\int_{B_{1}} d^{\mu} \leq C \quad \text { y } \quad \int_{B_{1}} d^{-\frac{\mu}{p-1}} \leq C .
$$

But, it is easy to see that $d(x) \simeq d\left(x, x_{F}\right)$ for all $x \in B \backslash B_{1}$. Therefore,

$$
\begin{aligned}
\int_{B} d^{\mu} & =\int_{B_{1}} d^{\mu}+\int_{B \backslash B_{1}} d^{\mu} \leq C\left(1+\int_{B \backslash B_{1}}\left|x-x_{F}\right|^{\mu}\right) \\
& \leq C\left(1+\int_{2 \operatorname{diam} F}^{r_{B}} \rho^{\mu} \rho^{n-1}\right) \leq C r_{B}^{\mu+n} .
\end{aligned}
$$

Analogously we can show that

$$
\int_{B} d^{-\frac{\mu}{p-1}} \leq C r_{B}^{-\frac{\mu}{p-1}+n}
$$

and therefore,

$$
\left(\frac{1}{|B|} \int_{B} d^{\mu}\right)\left(\frac{1}{|B|} \int_{B} d^{-\frac{\mu}{p-1}}\right)^{p-1} \leq C \frac{1}{|B|^{p}} r_{B}^{\mu+n} r_{B}^{-\mu+n(p-1)} \leq C .
$$

and the lemma is proved.

\section{REFERENCES}

[ADL] G. Acosta, R. G. Durán And A. Lombardi, Weighted Poincaré and Korn inequalities for Hölder $\alpha$ domains, Math. Meth. Appl. Sci. (MMAS) 29 (4), 387-400, 2006.

[AADL] G. Acosta, María G. Armentano, R. G. Durán and A. Lombardi, Nonhomogeneous Neumann problem for the Poisson equation in domains with an external cusp, Journal of Mathematical Analysis and Applications 310, pp 397-411, 2005.

[ADM] G. Acosta, R. G. Durán And M. A. Muschietti, Solutions of the divergence operator on John Domains, Advances in Mathematics 206(2), 373-401, 2006.

[ASV] D. N. ARnold, L. R. ScOtT And M. Vogelius, Regular inversion of the divergence operator with Dirichlet boundary conditions on a polygon, Ann. Scuola Norm. Sup. Pisa Cl. Sci-Serie IV XV, pp. 169-192, 1988.

[B] M. E. BogovskiI, Solution of the first boundary value problem for the equation of continuity of an incompressible medium, Soviet Math. Dokl. 20, pp. 1094-1098, 1979.

[BB] J. Bourgain And H. Brezis, On the equations div $Y=f$ and application to control of phases, Journal of the American Mathematical Society 16(2), pp. 393-426, 2002.

[Br] J. H. Bramble, A proof of the inf-sup condition for the Stokes equations on Lipschitz domains. Dedicated to Jim Douglas, Jr. on the occasion of his 75th birthday. Math. Models Methods Appl. Sci. 13 (2003), no. $3,361-371$.

[BS] S. C. Brenner and L. R. Scott, The Mathematical Theory of Finite Element Methods, SpringerVerlag, Berlin, 1994.

[BF] F. Brezzi And M. Fortin, Mixed and Hybrid Finite Element Methods, Springer Verlag, 1991.

[BK] S. M. Buckley And P. Koskela, New Poincaré inequalities from old, Annales Academiae Scientiarum Fennicae Mathematica 23, pp. 251-260, 1998.

[CM] M. Costabel And A. MCintosh, On Bogovskii and regularized Poincar integral operators for de Rham complexes on Lipschitz. 
[Da] M. DAuge Strongly elliptic problems near cuspidal points and edges. Partial differential equations and functional analysis, 93-110, Progr. Nonlinear Differential Equations Appl., 22, Birkhuser Boston, Boston, MA, 1996

[De] J. Detraz Classes de Bergman de fonctions harmoniques, Bull. Soc. Math. France 109, 259-268, 1981.

[Du1] R. G. DuRÁn The inf-sup condition for the Stokes equations: A constructive approach in general domains, Mathematisches Forschungsinstitut Oberwolfach, Workshop on Gemischte und nicht-standard Finite-Elemente-Methoden mit Anwendungen, Extended abstract, Report No. 5, pp. 270-272, 2005.

[Du2] R. G. Durán, Mixed Finite Element Methods, Mixed Finite Elements, Compatibility Conditions, and Applications, Lecture Notes in Mathematics, LNMCIME 1939, D. Boffi, L. Gastaldi, eds., Springer Verlag, 2008.

[DM1] R. G. Durán And M. A. Muschietti, On the traces of $W^{2, p}(\Omega)$ for a Lipschitz domain, Revista Matemática Complutense XIV(2), 371-377, 2001.

[DM2] R. G. DurÁn AND M. A. Muschietti, An explicit right inverse of the divergence operator which is continuous in weighted norms, Studia Math. 148 (3), 2001. 207-219.

[DST] R. G. Durán, M. Sanmartino And M.Toschi, Weighted a priori estimates for the Poisson equation, to appear in Indiana University Math. Journal.

[E] L. C. Evans, Partial Differential Equations, volume 19 of Graduate Studies in Mathematics, American Mathemaical Society, Providence, RI, 1998.

[Fr] K. O. FRIEDRICHS, On certain inequalities and characteristic value problems for analytic functions and for functions of two variables, Trans. Amer. Math. Soc., 41, pp. 321-364, 1937.

[Ga] M. Gagliardo, Caratterizzazioni delle tracce sulla frontieri relative ad alcune classi di funzioni in $n$ variabili, Ren. Sem. Mat. Univ. Padova 27, pp. 284-305, 1957.

[GHH] M. Geiert, H. Heck, AND M. Hieber, On the equation div $u=g$ and Bogovskii's operator in Sobolev spaces of negative order. Partial differential equations and functional analysis, 113-121, Oper. Theory Adv. Appl., 168, Birkhuser, Basel, 2006.

[GG] G. Geymonat, G. Gilardi, Contre-exemples á l'inégalité de Korn et au Lemme de Lions dans des domaines irréguliers, Equations aux Dérivées Partielles et Applications, Gauthiers-Villars, pp. 541-548, 1998.

[GK] G. Geymonat and F. Krasucki, On the existence of the Airy function in Lipschitz domains. Application to the traces of $H^{2}$, C. R. Acad. Sci. Paris, t. 330, serie 1, pp. 355-360, 2000.

[GT] D. Gilbarg and N. S. Trudinger, Elliptic Partial Differential equations of Second Order, Springer, Berlin, 1983.

[GR] V. Girault and P. A. Raviart, Finite Element Methods for Navier-Stokes Equations, Springer-Verlag, Berlin, 1986.

[Gi] P. Grisvard, Elliptic Problems in Nonsmooth Domains, Pitman, Boston, 1985.

[H] P. Harjulehto, Traces and Sobolev extension domains, Proc. Amer. Math. Soc., 134 (2006), 2373-2382.

[HP] C. O. Horgan And L. E. Payne, On inequalities of Korn, Friedrichs and Babuska-Aziz, Arch. Rational Mech. Anal., 82, pp. 165-179, 1983.

[MV] O. Martio and M. Vuorinen, Whitney cubes, p-capacity, and Minkowski content, Exposition. Math., $5(1)$, pp. 17-40, 1987.

[KO] V. A. Kondratiev and O. A. Oleinik, On Korn's inequalities, C. R. Acad. Sci. Paris, 308, pp. 483-487, 1989.

[K] A. Kufner, Weighted Sobolev Spaces, John Wiley \& Sons, Inc., New York, 1985.

[KuOp] A. Kufner And B. OpIC, Hardy type inequalities, University of West Bohemia Pilsen, 1992.

[MP1] V. G. MaZ'ya And S. V. Poborchi, On the solvability of the Neumann problem for a planar domain with a peak. Vestnik St. Petersburg Univ. Math. 41 (2008), no. 2, 145-160.

[MP2] V. G. MaZ'ya AND S. V. PobORChi, On solvability of the Neumann problem in an energy space for a domain with peak. Georgian Math. J. 14 (2007), no. 3, 499-518.

[MP3] V. G. MaZ'ya And S. V. PoBorchi, Theorems for embedding Sobolev spaces on domains with a peak and on Hlder domains. (Russian) Algebra i Analiz 18 (2006), no. 4, 95-126; translation in St. Petersburg Math. J. 18 (2007), no. 4, 583-605.

[N] S. A. NAZARov, On the eigenoscillations of an elastic body with a blunted peak. (Russian) Dokl. Akad. Nauk 416 (2007), no. 4, 481-485.

[NT] S. A. NaZarov and J. TASkinen, On the spectrum of the Steklov problem in a domain with a peak. Vestnik St. Petersburg Univ. Math. 41 (2008), no. 1, 45-52.

[S] E. M. Stein, Singular Integrals and Differentiability Properties of Functions, Princeton Univ. Press, 1970 .

[S2] E. M. Stein, Harmonic Analysis. Real Variable Methods, Orthogonality, and Oscillatory Integrals, Princeton Univ. Press, 1993.

[T] R. Temam, Navier-Stokes Equations, North-Holland, Amsterdam, 1984. 
Departamento de Matemática, Facultad de Ciencias Exactas y Naturales, Universidad de Buenos Aires, 1428 Buenos Aires, Argentina.

E-mail address: rduran@dm.uba.ar

Departamento de Matemática, Facultad de Ciencias Exactas y Naturales, Universidad de Buenos Aires, 1428 Buenos Aires, Argentina.

E-mail address: flopezg@dm.uba.ar 